\title{
Matéria orgânica nas frações granulométricas de um latossolo vermelho distroférrico sob diferentes sistemas de uso e manejo
}

\section{Soil organic matter in the granulometric fractions of an oxissol under different uses and management systems}

\author{
Carmen Silvia Vieira Janeiro Neves ${ }^{1 *}$; Christian Feller ${ }^{2}$; \\ Marie-Christine Larré-Larrouy ${ }^{3}$
}

Resumo

\begin{abstract}
A matéria orgânica do solo é um importante componente da sustentabilidade dos sistemas agrícolas. Efetuouse o fracionamento granulométrico da matéria orgânica de um Latossolo Vermelho distroférrico argiloso, do Norte do Paraná, sob pomar de citros manejado (por nove anos) com: a) cobertura de leguminosa (Arachis prostrata); b) vegetação espontânea, mantida por grade e roçadora; c) sem vegetação intercalar (capinado). O mesmo solo foi estudado também sob culturas anuais (preparo convencional) por vinte anos e floresta nativa. A cobertura com leguminosa e a manutenção da vegetação espontânea no solo do pomar aumentaram os estoques de $\mathrm{C}$ e $\mathrm{N}$ do solo em comparação ao sistema sem vegetação intercalar e ao solo sob culturas anuais. Nos sistemas de uso e manejo avaliados, as maiores proporções dos estoques de $\mathrm{C}$ e $\mathrm{N}$ do solo encontraram-se associadas à fração argila $(0-2 \mu \mathrm{m}), \operatorname{com} 35 \mathrm{a} 51 \%$ do total, e à fração silte $(2-20 \mu \mathrm{m})$, com 13 a $30 \%$. O estoque de carbono ( $\mathrm{em} \mathrm{Mg} \mathrm{Cha-1)}$ na profundidade $0-10 \mathrm{~cm}$ foi reduzido em $33 \%$, com culturas anuais, e em $19 \%$, no pomar capinado, em relação à floresta nativa; e foi ligeiramente aumentado nas condições de pomar com cobertura vegetal. As maiores perdas de $\mathrm{C}$ e $\mathrm{N}$ ocorreram nas frações areia grossa + resíduos vegetais $(50-2.000 \mu \mathrm{m}) \mathrm{e}$ areia fina $(50-200 \mu \mathrm{m})$. No pomar com leguminosa, quando comparado com culturas anuais, a recuperação da matéria orgânica foi maior na fração areia muito fina (20-50 $\mu \mathrm{m})$.

Palavras-chave: Carbono, nitrogênio, estoque, frações granulométricas, solos tropicais
\end{abstract}

\begin{abstract}
Soil organic matter (SOM) is an important component of agriculture sustainability. A clayey oxisol was studied by SOM particle size fractions in a citrus orchard managed (for a 9 year period) with a) Arachis prostrata ground cover; b) spontaneous vegetation controlled by mowing and tillage; c) bare soil. Also the same soil was studied under a 20 year period of annual crops (conventional tillage) and native forest. The orchard ground covers increased the soil $\mathrm{C}$ and $\mathrm{N}$ stocks when compared to orchard bare soil and annual crops. For all situations, the largest proportion of the SOM was found in the clay fraction (0-2

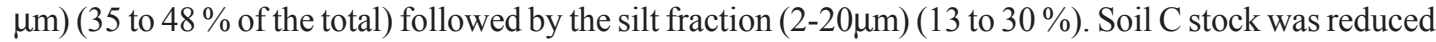
by $33 \%$ with annual crops and by $19 \%$ with bare soil orchard, and was slightly higher with orchard ground covers, when compared to native forest. The carbon losses in the layer $0-10 \mathrm{~cm}$ were 23 to $45 \%$ comparing the native forest to the crops, being larger with annual crops for 20 years and smaller at orchard with $A$. prostrata ground cover. The greatest SOM losses were in the 50-2,000 $\mu \mathrm{m}$ (vegetal debris + coarse sand), and fine sand $(50-200 \mu \mathrm{m})$ fractions. The greater recuperation of SOM in the orchard with leguminous cover was in the 20-50 $\mathrm{m}$ fraction when compared to annual crops.
\end{abstract}

Key words: Carbon, nitrogen, stock, particle size fractions, tropical soils

1 Eng. Agr ${ }^{\text {a. }}$, Dra., Professora do Dep. de Agronomia, Universidade Estadual de Londrina, C.P. 6001, CEP 86.051-990, Londrina, PR. Bolsista do CNPq. E-mail: csvjneve@uel.br - Autora para correspondência

2 Pedólogo, Dr., IRD/MOST - B.P. 64501, 34394, Montpellier, França. E-mail: feller@mpl.ird.fr

3 Química, IRD/MOST - B.P. 64501, 34394, Montpellier, França

* Autor para correspondência

Recebido para publicação 16/06/04 Aprovado em 10/12/04 


\section{Introdução}

A fertilidade dos solos tem estreita relação com seu teor de matéria orgânica, a qual influencia diversas propriedades biológicas, químicas e físicas do solo, e auxilia na redução do processo de erosão (VAUGHAN; ORD, 1985; SÁ, 1993). Além disso, sistemas agrícolas que favoreçam o seqüestro de carbono no solo são vistos como alternativa para mitigar o aumento das emissões de $\mathrm{CO}_{2}$ na atmosfera (LAL, 1997). Dessa forma, é importante monitorar o efeito de práticas de manejo e diferentes formas de uso da terra em relação ao seu efeito nos ciclos de $\mathrm{C}$ e $\mathrm{N}$ do solo.

Existe uma estreita relação entre a matéria orgânica (MO) do solo e a fração mineral do solo, formando complexos organo-minerais (SCHNITZER, 1986). Solos com maior conteúdo de argila têm maior poder de conservação e estabilização da MO (LEPSCH; SILVA; ESPIRONELO, 1982). Porém, devido à complexidade da associação organo-mineral, o estudo da MO do solo tem sido feito, tradicionalmente, de acordo com um enfoque bioquímico, procurando-se minimizar o conteúdo de cinzas, enquanto que o estudo dos minerais é feito, geralmente, depois da eliminação da MO (CHRISTENSEN, 1992). O fracionamento físico do solo, de acordo com o tamanho das partículas ou por densidade, tem se mostrado uma ferramenta útil no estudo da MO do solo, revelando diferenças tanto na estrutura como na dinâmica da MO quando esta se encontra ligada às partículas de diferentes tamanhos (FELLER; BEARE, 1997). Muitos trabalhos têm demonstrado que quando o teor total de carbono do solo muda, as concentrações de carbono associado às partículas de tamanhos diferentes são afetadas de forma diversa, indicando que a MO do solo tem períodos diferenciados de "turnover", dependendo da partícula à qual ela se liga (CERRI et al., 1985).

O objetivo deste trabalho foi avaliar os teores de MO de um latossolo argiloso sob pomar, manejado durante nove anos com leguminosa permanente, vegetação espontânea controlada por meio de grade/ roçadeira ou mantido descoberto por meio de capina, com os teores de MO do mesmo solo cultivado com lavouras anuais sob preparo convencional. Objetivouse, também, identificar quais as frações da MO tiveram relação com as alterações ocorridas nos teores totais de carbono, buscando-se alternativas de manejo que favoreçam o seqüestro de carbono.

\section{Material e Métodos}

O trabalho foi realizado em Londrina (PR), cujo clima é do tipo Cfa, subtropical úmido, com chuvas em todas as estações, podendo ocorrer seca no período de inverno. A temperatura média anual é de $20,7^{\circ} \mathrm{C}$ e a precipitação anual é de $1.615 \mathrm{~mm}$. Pela análise granulométrica, o solo estudado (Latossolo Vermelho distroférrico) apresentou $691 \mathrm{~g} \mathrm{~kg}^{-1} \mathrm{de}$ argila, $216 \mathrm{~g} \mathrm{~kg}^{-1}$ de silte e $93 \mathrm{~g} \mathrm{~kg}^{-1}$ de areia. A análise química do solo (EMBRAPA, 1979), realizada por ocasião da instalação do pomar, revelou os seguintes valores (camada de 0-15 cm): $\mathrm{pH}\left(\mathrm{H}_{2} \mathrm{O}\right)=5,38$; $\mathrm{MO}$ $=29,2 \mathrm{~g} \mathrm{dm}^{-3} ; \mathrm{P}($ Mehlich-1 $)=2,04 \mathrm{mg} \mathrm{dm}^{-3} ; \mathrm{K}^{+}=$ $2,3 \mathrm{mmol}_{\mathrm{c}} \mathrm{dm}^{-3} ; \mathrm{Ca}^{+2}=45 \mathrm{mmol}_{\mathrm{c}} \mathrm{dm}^{-3} ; \mathrm{Mg}^{+2}=19$ $\mathrm{mmol}_{\mathrm{c}} \mathrm{dm}^{-3} ; \mathrm{H}^{+}+\mathrm{Al}^{3+}=70 \mathrm{mmol}_{\mathrm{c}} \mathrm{dm}^{-3}$. Para a camada de 15-30 cm, os resultados foram: $\mathrm{pH}\left(\mathrm{H}_{2} \mathrm{O}\right)$ $=5,02 ; \mathrm{MO}=17,5 \mathrm{~g} \mathrm{dm}^{-3} ; \mathrm{P}($ Mehlich-1) $=0,92 \mathrm{mg}$ $\mathrm{dm}^{-3} ; \mathrm{K}^{+}=1,1 \mathrm{mmol}_{\mathrm{c}} \mathrm{dm}^{-3} ; \mathrm{Ca}^{+2}=33 \mathrm{mmol}_{\mathrm{c}} \mathrm{dm}^{-3}$; $\mathrm{Mg}^{+2}=32 \mathrm{mmol}_{\mathrm{c}} \mathrm{dm}^{-3} ; \mathrm{H}^{+}+\mathrm{Al}^{3+}=61 \mathrm{mmol}_{\mathrm{c}} \mathrm{dm}^{-3}$.

$\mathrm{Na}$ área do pomar de citrus, desmatada cerca de 50 anos antes e cultivada com café seguido de mandioca, foi conduzida durante nove anos um experimento de manejo de solo, nas entrelinhas das plantas cítricas (NEVES et al., 1998). O pomar recebeu calcário dolomítico a cada 3-4 anos e adubação mineral (sulfato de amônio, superfosfato simples e cloreto de potássio) com base nos teores dos elementos no solo e nas produções médias anuais. Para o estudo da MO do solo foram utilizados os seguintes tratamentos: $\mathrm{C}$, capina manual o ano inteiro; VE, vegetação espontânea (predomínio de gramíneas) controlada com uma gradagem a disco (no período seco) e roçadora (três a quatro vezes no período de 
chuvas); A, cobertura verde com a leguminosa perene Arachis prostrata Benth. Amostras do mesmo solo também foram retiradas, em área adjacente ao experimento, sob floresta tropical subperenifólia nativa (FN) e sob cultura anual (CA) manejada por 20 anos em sistema de preparo convencional, com lavração e gradagem duas vezes ao ano (na safra em questão cultivou-se aveia no inverno após algodão no verão). As amostragens foram realizadas a $0-10$ e $10-20 \mathrm{~cm}$ de profundidade.

A metodologia utilizada para o fracionamento granulométrico da MO do solo foi a descrita por Feller et al. (1991) e Gavinelli et al. (1995). O estudo foi realizado em amostras simples coletadas em quatro repetições, dos tratamentos $\mathrm{A}$ e $\mathrm{C}$ e também em amostras compostas dos demais sistemas de manejo, resultantes de seis subamostras coletadas a campo. Utilizaram-se amostras de $10 \mathrm{~g}$ de solo peneirado a 0-2 mm. Cada amostra foi misturada a $200 \mathrm{~mL}$ de água e submetida a agitação rotativa $(50 \mathrm{rpm})$ por duas horas com hexametafosfato de sódio $(0,5 \mathrm{~g} /$ amostra) e bolas de ágata. As frações 200-2.000 $\mu \mathrm{m}$ e $50-200 \mu \mathrm{m}$ foram obtidas por peneiramento com água destilada. Para facilitar a dispersão na obtenção das frações $<50 \mu \mathrm{m}$, utilizou-se ultrasom (10 minutos, 600 watts, $90 \%$ de potência, interrupções automáticas de $30 \%$ do tempo total, com energia total de $150 \mathrm{~J} \mathrm{~mL}^{-1}$ ) (BALESDENT ; PETRAUD ; FELLER, 1991; ROSCOE; BUURMAN ; VELTHORST, 2000). Em seguida, a fração 20-50 $\mu \mathrm{m}$ foi obtida por peneiramento lavando-se com água destilada. A fração $0-20 \mu \mathrm{m}$ foi transferida para proveta de $1000 \mathrm{~mL}$, cujo volume foi completado com água e agitada manualmente com 30 rotações verticais, em aproximadamente 30 segundos. Imediatamente, uma primeira alíquota de $200 \mathrm{~mL}$ foi tomada da suspensão, com auxílio de pipeta a $10 \mathrm{~cm}$ da superfície, constituindo a fração 0-20 $\mu \mathrm{m}$. Após repouso de 8 horas, a $20{ }^{\circ} \mathrm{C}$, foi sifonada uma segunda alíquota, representando a fração $<2 \mu \mathrm{m}$ (GAVINELLI et al., 1995). Estas duas frações amostradas foram floculadas com $1 \mathrm{~mL}$ de uma solução saturada de $\mathrm{SrCl}_{2}$, centrifugadas e lavadas no frasco de centrifugação e novamente centrifugadas. Todas as frações obtidas foram levadas à estufa até peso constante $\left(60^{\circ} \mathrm{C}\right)$ e moídas a $200 \mu \mathrm{m}$. O cálculo da proporção de cada fração foi feito determinando-se a percentagem em relação à massa total da amostra de solo. A fração 2-20 $\mu \mathrm{m}$ foi obtida por diferença. Os teores de $\mathrm{C}$ e $\mathrm{N}$, no solo não fracionado e nas frações granulométricaso, foram determinados com o Autoanalisador Elementar LECO CHN 600 (Carlo Erba). O conteúdo de $\mathrm{C}$ e $\mathrm{N}$ das frações (em $\mathrm{mg} \mathrm{g}^{-1}$ de solo) foi calculado considerando-se a massa de cada fração. O estoque de carbono ( $\mathrm{em} \mathrm{Mg} \mathrm{ha}^{-1}$ ) foi calculado, considerandose a camada de solo amostrada e a densidade do solo, avaliada pelo método do anel volumétrico.

Foram calculados a média, o desvio padrão e o coeficiente de variação da massa de solo e dos teores de $\mathrm{C}$ e $\mathrm{N}$ das amostras simples, com o objetivo de verificar a variabilidade dos dados.

\section{Resultados e Discussão}

Teores e estoques de $C$ e $N$ no solo não fracionado (NF)

Foram observadas maiores amplitudes nos teores de $\mathrm{C}$ e $\mathrm{N}$ do solo entre os sistemas de manejo na camada $0-10 \mathrm{~cm}$ do que na camada $10-20 \mathrm{~cm}$ de profundidade (Tabelas 1 e 2). O solo, originalmente com cerca de $32 \mathrm{mg} \mathrm{C} \mathrm{g}^{-1}$ (sob floresta), quando submetido a cultivos anuais teve uma redução de cerca de $50 \%$ do teor de C, passando a teores de 17 $\mathrm{mg} \mathrm{C} \mathrm{g}^{-1}$. A diminuição de $\mathrm{N}$ foi de cerca de $36 \%$. Reduções semelhantes foram encontradas por Feller (1995) e Freixo et al. (2002). Entretanto, Sá et al. (2001) observaram que o sistema de plantio direto aumentou significativamente a MO do solo, quando comparado com a vegetação de campo nativo.

Para os tratamentos usados no pomar, o manejo utilizando coberturas vegetais (leguminosa ou vegetação nativa, com predomínio de gramíneas) possibilitou maiores teores totais de $\mathrm{C}$ em relação ao solo com culturas anuais, chegando a teores de 25 
mg C g ${ }^{-1}$ representado cerca de $80 \%$ do teor de $\mathrm{C}$ do solo de floresta nativa (Figura 1). Mas quando o pomar foi mantido com o solo descoberto por meio de capinas, o teor de $\mathrm{C}$ foi de $18 \mathrm{mg} \mathrm{g}^{-1}$, equivalente ao do solo com culturas anuais.

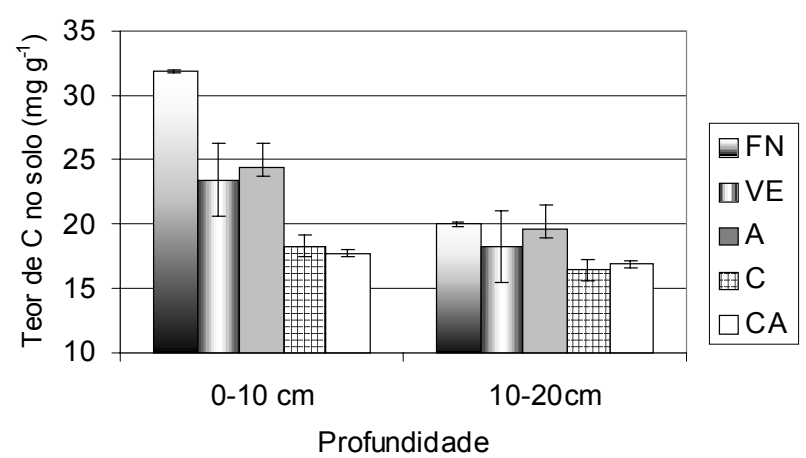

Figura 1. Teor de C do solo não fracionado (em mg g-1 de solo) para floresta nativa (FN), pomar com vegetação espontânea (VE), pomar com A. prostrata (A), pomar capinado $(\mathrm{C})$ e culturas anuais $(\mathrm{CA})$, nas profundidades de $0-10 \mathrm{~cm}$ e $10-20 \mathrm{~cm}$.

$\mathrm{O}$ teor de $\mathrm{N}$ do solo também aumentou com coberturas vegetais no pomar, sendo a recuperação maior para a cobertura com leguminosa, beneficiada pela fixação biológica (Tabela 1). Desjardins et al. (1994) obtiveram teores semelhantes entre o $\mathrm{N}$ do solo sob floresta e sob pastagem, enquanto que Sá et al. (2001) tiveram aumento com a introdução de culturas devido à mineralização da biomassa da vegetação nativa.

Os estoques de $\mathrm{C}$ e $\mathrm{N}$ no solo não fracionado (Tabelas 1 e 2) foram menores nos tratamentos Capina e culturas anuais e nos tratamentos com cobertura vegetal espontânea e de Arachis no pomar apresentaram valores ligeiramente superiores ao apresentado pela floresta nativa, na camada $0-10 \mathrm{~cm}$, em razão da menor densidade do solo da floresta. $\mathrm{Na}$ camada 10-20 $\mathrm{cm}$ todas as situações apresentaram maior estoque do que a vegetação de mata nativa. Este aumento em profundidade com a retirada da mata e a introdução de culturas foi observado também por Cerri, Feller e Chauvel (1991) com cana-de-açúcar e por Feigl, Melillo e Cerri (1995) com pastagem.
$\mathrm{O}$ estoque de $\mathrm{C}$ no solo sob floresta na camada de $0-10 \mathrm{~cm}$ foi de $27,12 \mathrm{Mg} \mathrm{ha}^{-1}$, enquanto que para a camada 10-20 cm foi de 19,0 $\mathrm{Mg} \mathrm{ha}^{-1}$, concordando com o verificado por Cerri et al. (1991), que constataram um brusco decréscimo do estoque de $\mathrm{C}$ a partir da profundidade de $6 \mathrm{~cm}$ em solo de floresta. Os valores de estoque de $\mathrm{C}$ aqui encontrados foram superiores aos observados por Desjardins et al. (1994) que obtiveram 19,2 $\mathrm{Mg} \mathrm{ha}^{-1}$, em um latossolo podzolisado sob floresta tropical na Amazônia, o que pode ser explicado pelo menor teor de argila mais silte daquele solo $(27,4 \%)$, enquanto que o solo da presente pesquisa apresenta $90,7 \%$ de argila mais silte. Existe uma relação linear positiva entre o teor de argila mais silte e o teor de MO do solo, principalmente para os oxisolos, provavelmente devido à proteção conferida pela argila à ação dos microorganismos (FELLER; BEARE, 1997).

O uso de plantas de cobertura no pomar (tanto leguminosas como gramíneas) manteve a $\mathrm{MO}$ em níveis mais elevados do que o solo descoberto através de capina permanente, favorecendo a sustentabilidade do sistema produtivo, apesar da produção do pomar não apresentar diferenças significativas (NEVES et al., 1998). Com isso, um pomar bem manejado do ponto de vista da cobertura do solo pode ser indicado como uma forma de recuperação de áreas degradadas, apresentando ainda a vantagem de gerar renda com a produção de frutas.

\section{Frações granulométricas da matéria orgânica}

\section{Aspectos Metodológicos}

Devido à textura muito argilosa do solo estudado, a fração mais importante, representando até $69 \%$ da massa do solo, é a argila $(<2 \mu \mathrm{m})$. Nessa fração, e também na fração silte $(2-20 \mu \mathrm{m})$, que representa $23 \%$ da massa de solo, os coeficientes de variação (CV) foram baixos (GOMES, 1987), de 0,9 a 4,8 \% (Tabela 3), o que evidencia a alta precisão do método utilizado, semelhante ao observado por Freitas et al. (2000). Os maiores valores de CV obtidos para as 
Tabela 1. Características das frações granulométricas da matéria orgânica do solo sob diferentes usos e manejos, na profundidade de $0-10 \mathrm{~cm}$.

\begin{tabular}{|c|c|c|c|c|c|c|c|c|c|c|c|}
\hline \multirow[t]{2}{*}{ Fração } & \multirow[t]{2}{*}{ Massa } & \multicolumn{6}{|c|}{ Densid. Estoque } & $\mathrm{N}$ & & $\begin{array}{c}\text { Estoque } \\
\mathrm{N}\end{array}$ & \multirow[t]{2}{*}{$\begin{array}{l}\text { Rel. } \\
\text { C/N }\end{array}$} \\
\hline & & $\begin{array}{l}\mathrm{mg} \mathrm{g}^{-1} \\
\text { fração }\end{array}$ & $\begin{array}{c}\mathrm{mg} \mathrm{g}^{-1} \\
\text { solo }\end{array}$ & do total & $\mathrm{g} \mathrm{cm}^{-3}$ & $\mathrm{Mg} \mathrm{ha}^{-1}$ & $\begin{array}{l}\mathrm{mg} \mathrm{g}^{-1} \\
\text { fração }\end{array}$ & $\begin{array}{c}\mathrm{mg} \mathrm{g}^{-1} \\
\text { solo }\end{array}$ & $\begin{array}{c}\% \\
\text { do total }\end{array}$ & $\mathrm{Mg} \mathrm{ha}^{-1}$ & \\
\hline \multicolumn{12}{|c|}{ FN - floresta nativa } \\
\hline $200-2.000$ & 2,34 & 118,1 & 2,8 & 10,32 & 0,85 & 2,35 & 9,49 & 0,22 & 8,40 & 0,19 & 12,5 \\
\hline $50-200$ & 12,59 & 37,4 & 4,7 & 17,55 & 0,85 & 4,00 & 3,49 & 0,44 & 16,65 & & 10,7 \\
\hline $20-50$ & 2,94 & 27,2 & 0,8 & 2,98 & 0,85 & 0,68 & 2,05 & 0,06 & 2,27 & & 13,3 \\
\hline $2-20$ & 20,81 & 36,1 & 7,5 & 28,02 & 0,85 & 6,39 & 3,61 & 0,75 & 28,38 & & 10,0 \\
\hline $0-2$ & 61,31 & 18,0 & 11,0 & 41,13 & 0,85 & 9,38 & 1,91 & 1,17 & 44,31 & 1, & 9,4 \\
\hline Total & 100,0 & & 26,8 & 100,00 & 0,85 & 22,81 & & 2,64 & 100,00 & 2, & 10,2 \\
\hline & 100,0 & & 31,9 & & 0,85 & 27,12 & & 3,09 & & 2, & 10,3 \\
\hline \multicolumn{12}{|c|}{ VE - vegetação espontânea (pomar) } \\
\hline $200-2.000$ & 1,61 & 71,0 & 1,2 & 5,33 & 1,26 & 1,45 & 3,66 & 0,06 & 2,99 & 0,07 & 19,5 \\
\hline $50-200$ & 9,23 & 29,4 & 2,7 & 12,61 & 1,26 & 3,43 & & 0,19 & 72 & & 14,2 \\
\hline $20-50$ & 3,43 & 15,0 & 0,5 & & 1,26 & & & 0,03 & & & 15,9 \\
\hline & 20,18 & 34,3 & 6,9 & 32,13 & 1,26 & 8,73 & 3,03 & 0,61 & 30,94 & & 11,3 \\
\hline $0-2$ & 68,86 & 14,9 & 10,3 & 47,57 & 1,26 & 12,93 & 1,57 & 1,08 & 54,73 & & 9,5 \\
\hline & 103,32 & & 21,6 & 100,00 & 1,26 & 27,18 & & 1,98 & 100,00 & 2, & 10,9 \\
\hline & 100,0 & & 23,5 & & 1,26 & 29,57 & & 2,30 & & 2, & 10,2 \\
\hline \multicolumn{12}{|c|}{ A - Arachis prostrata (pomar) } \\
\hline $200-2.000$ & 0,97 & 102,4 & 1,0 & 4,57 & 1,22 & 1,21 & 6,91 & 0,07 & 3,18 & & 14,8 \\
\hline $50-200$ & 9,34 & 26,7 & 2,5 & 11,51 & 1,22 & 3,04 & & 0,20 & & & 12,6 \\
\hline $20-50$ & 3,53 & & 1,0 & & 1,22 & 1,23 & & 0,07 & & & 15,5 \\
\hline & 19 , & & 7,0 & 32 , & 1,22 & & & 0,63 & & & 11,1 \\
\hline $0-2$ & 68,9 & 14,8 & 10,2 & 47,13 & 1,22 & 12,44 & 1,67 & 1,15 & 54,65 & 0 & 8,9 \\
\hline & 97,83 & & 21,6 & 100,00 & 1,22 & 26,40 & & 2,11 & 100,00 & & 10,3 \\
\hline & 100,0 & & 24,5 & & 1,22 & 29,89 & & 2,52 & & 3, & 9,7 \\
\hline \multicolumn{12}{|c|}{ C - capina (pomar) } \\
\hline $200-2.000$ & 1,00 & 53,3 & 0,5 & 3,41 & 1,21 & 0,64 & 2,13 & 0,02 & 1,35 & & 25,2 \\
\hline & 8,72 & & 1,4 & & 1,21 & 1,73 & & 0,11 & & & 13,2 \\
\hline $20-5$ & 3,41 & 14,7 & 0,5 & & 1,21 & 0,61 & 0,96 & 0,03 & & & 15,2 \\
\hline & 21,28 & 25,2 & 5,4 & 34,4 & 1,21 & 6,49 & 2,48 & 0,53 & 33,98 & & 10,2 \\
\hline $0-2$ & 69,72 & 11,1 & 7,7 & 49,74 & 1,21 & 9,37 & 1,24 & 0,86 & & & 9,0 \\
\hline & 104,13 & & 15,6 & 100,00 & 1,21 & 18,83 & & 1,55 & 100,00 & & 10,0 \\
\hline & 100 & & 18,3 & & 1,21 & 22,14 & & 1,70 & & & 10,8 \\
\hline \multicolumn{12}{|c|}{ CA - culturas anuais } \\
\hline $200-2.000$ & 0,71 & 71,9 & 0,5 & 3,07 & 1,02 & 0,52 & & & & & 17,0 \\
\hline $50-200$ & 6,75 & 26,2 & 1,8 & 10,66 & 1,02 & 1,81 & 1,73 & 0,12 & 7,22 & & 15,1 \\
\hline $20-50$ & 3,92 & 14,4 & 0,6 & 3,37 & 1,02 & 0,57 & 0,93 & 0,03 & 2,04 & 0,03 & 17,0 \\
\hline $2-20$ & 20,82 & 25,2 & 5,3 & 31,63 & 1,02 & 5,36 & 2,32 & 0,48 & 29,73 & 0,49 & 10,9 \\
\hline $0-2$ & 71,54 & 11,9 & 8,5 & 51,27 & 1,02 & 8,68 & 1,34 & 0,96 & 59,16 & 0,98 & 8,9 \\
\hline Total & 103,74 & & 16,6 & 100,00 & 1,02 & 16,93 & & 1,62 & 100,00 & 1,65 & 10,2 \\
\hline solo NF & 100 & & 17,7 & & 1,02 & 18,05 & & 1,67 & & 1,70 & 10,6 \\
\hline
\end{tabular}

NF: não fracionado 
Tabela 2. Características das frações granulométricas da matéria orgânica do solo sob diferentes usos e manejos, na profundidade de $10-20 \mathrm{~cm}$.

\begin{tabular}{|c|c|c|c|c|c|c|c|c|c|c|c|}
\hline \multirow{2}{*}{$\begin{array}{l}\text { Fração } \\
\mu \mathrm{m}\end{array}$} & \multirow[t]{2}{*}{ Massa } & \multicolumn{3}{|c|}{$\mathrm{C}$} & \multirow{2}{*}{$\begin{array}{l}\text { Densid. } \\
\text { solo } \\
\mathrm{g} \mathrm{cm}^{-3}\end{array}$} & \multirow{2}{*}{$\begin{array}{l}\text { Estoque } \\
\mathrm{C} \\
\mathrm{Mg} \mathrm{ha}^{-1}\end{array}$} & \multicolumn{3}{|c|}{$\mathrm{N}$} & \multirow{2}{*}{$\begin{array}{c}\text { Estoque } \\
\mathrm{N} \\
\mathrm{Mg} \mathrm{ha}^{-1}\end{array}$} & \multirow{2}{*}{$\begin{array}{l}\text { Rel. } \\
\text { C/N }\end{array}$} \\
\hline & & $\begin{array}{l}\mathrm{mg} \mathrm{g}^{-1} \\
\text { fração }\end{array}$ & $\begin{array}{l}\mathrm{mg} \mathrm{g}^{-} \\
{ }_{\text {solo }}\end{array}$ & $\begin{array}{c}\% \\
\text { do total }\end{array}$ & & & $\begin{array}{l}\mathrm{mg} \mathrm{g}^{-1} \\
\text { fração }\end{array}$ & $\begin{array}{c}\mathrm{mg} \mathrm{g}^{-1} \\
\text { solo }\end{array}$ & $\begin{array}{c}\% \\
\text { do total } \\
\end{array}$ & & \\
\hline \multicolumn{12}{|c|}{ FN - floresta nativa } \\
\hline $200-2.000$ & 0,47 & 111,0 & 0,5 & 3,54 & 0,95 & 0,50 & 6,76 & 0,03 & 2,29 & 0,03 & 16,6 \\
\hline $50-200$ & 6,47 & 31,9 & 2,1 & 13,82 & 0,95 & 1,97 & 2,01 & 0,13 & 9,30 & 0,12 & 15,9 \\
\hline $20-50$ & 3,39 & 14,2 & 0,5 & 3,20 & 0,95 & 0,46 & 0,87 & 0,03 & 2,07 & 0,03 & 16,6 \\
\hline $2-20$ & 14,64 & 18,2 & 2,7 & 17,82 & 0,95 & 2,54 & 1,65 & 0,24 & 17,31 & 0,23 & 11,0 \\
\hline $0-2$ & 70,97 & 13,0 & 9,2 & 61,62 & 0,95 & 8,77 & 1,36 & 0,97 & 69,03 & 0,92 & 9,6 \\
\hline Total & 95,95 & & 15,0 & 100,00 & 0,95 & 14,23 & & 1,40 & 100,00 & 1,33 & 10,7 \\
\hline solo NF & 100,00 & & 20,0 & & 0,95 & 19,00 & & 1,74 & & 1,66 & 11,5 \\
\hline \multicolumn{12}{|c|}{ VE - vegetação espontânea (pomar) } \\
\hline $200-2.000$ & 1,09 & 54,5 & 0,6 & 3,55 & 1,21 & 0,71 & 1,77 & 0,02 & 1,11 & 0,02 & 31,1 \\
\hline $50-200$ & 7,77 & 13,1 & 1,0 & 6,14 & 1,21 & 1,23 & 0,86 & 0,07 & 3,92 & 0,08 & 15,2 \\
\hline $20-50$ & 3,56 & 17,6 & 0,6 & 3,79 & 1,21 & 0,76 & 1,08 & 0,04 & 2,22 & 0,05 & 16,6 \\
\hline $2-20$ & 21,88 & 23,6 & 5,2 & 31,05 & 1,21 & 6,24 & 2,33 & 0,51 & 29,75 & 0, & 10,1 \\
\hline $0-2$ & 70,91 & 13,0 & 9,2 & 55,48 & 1,21 & 11,16 & 1,52 & 1,08 & 63,00 & 1,30 & 8,6 \\
\hline Total & 105,23 & & 16,6 & 100,00 & 1,21 & 20,11 & & 1,71 & 100,00 & 2,0 & 9,7 \\
\hline solo NF & 100,00 & & 18,2 & & 1,21 & 22,05 & & 1,80 & & 2,1 & 10,1 \\
\hline \multicolumn{12}{|c|}{ A - Arachis prostrata (pomar) } \\
\hline $200-2.000$ & 0,88 & 65,5 & 0,6 & 2,95 & 1,25 & 0,73 & 3,02 & 0,03 & 1,61 & 0,03 & 21,5 \\
\hline $50-2$ & 8,39 & 13,8 & 1,2 & 5,91 & 1,25 & 1,45 & 0,99 & 0,08 & 4,94 & 0,10 & 14,0 \\
\hline $20-50$ & 3,57 & 18,8 & 0,7 & 3,41 & 1,25 & 0,84 & 1,16 & 0,04 & 2,44 & 0,05 & 16,3 \\
\hline $2-20$ & 19,49 & 25,7 & 5,0 & 25,57 & 1,25 & 6,28 & 2,24 & 0,44 & 25,94 & 0,5 & 11,5 \\
\hline $0-2$ & 70,06 & 13,8 & 9,7 & 2,95 & 1,25 & 12,09 & 1,55 & 1,09 & 65,08 & 1,3 & 8,8 \\
\hline Total & 102,39 & & 17,1 & 100,00 & 1,25 & 21,38 & & 1,68 & 100,00 & 2,10 & 10,2 \\
\hline solo NF & 100,00 & & 19,6 & & 1,25 & 24,54 & & 1,84 & & 2,3 & 10,7 \\
\hline \multicolumn{12}{|c|}{ C - capina (pomar) } \\
\hline $200-2.000$ & 0,75 & 34,7 & 0,3 & 1,77 & 1,22 & 0,32 & 1,36 & 0,01 & 0,66 & 0,01 & 26,0 \\
\hline $50-200$ & 7,69 & 14,2 & 1,1 & 7,43 & 1,22 & 1,33 & 0,92 & 0,07 & 4,68 & 0,0 & 15,4 \\
\hline $20-50$ & 3,43 & 8,1 & 0,3 & 1,91 & 1,22 & 0,34 & 0,54 & 0,02 & 1,25 & 0,02 & 14,7 \\
\hline $2-20$ & 19,62 & 24,5 & 4,8 & 32,83 & 1,22 & 5,88 & 2,26 & 0,44 & 28,99 & 0,54 & 11,0 \\
\hline $0-2$ & 70,38 & 11,7 & 8,2 & 56,06 & 1,22 & 10,04 & 1,39 & 0,98 & 64,43 & 1,19 & 8,4 \\
\hline Total & 101,87 & & 14,7 & 100,00 & 1,22 & 17,91 & & 1,52 & 100,00 & 1,85 & 9,7 \\
\hline solo NF & 100,00 & & 16,5 & & 1,22 & 20,09 & & 1,75 & & 2,13 & 9,4 \\
\hline \multicolumn{12}{|c|}{ CA - culturas anuais } \\
\hline $200-2.000$ & 0,72 & 64,3 & 0,5 & 3,21 & 1,18 & 0,54 & 3,00 & 0,02 & 1,60 & 0,03 & 20,9 \\
\hline $50-200$ & 5,73 & 17,5 & 1,0 & 6,98 & 1,18 & 1,1 & 1,06 & 0,06 & 4,42 & 0,0 & 16,4 \\
\hline $20-50$ & 3,29 & 13,9 & 0,5 & 3,21 & 1,18 & 0,54 & 0,86 & 0,03 & 2,03 & 0,03 & 16,4 \\
\hline $2-20$ & 18,44 & 23,5 & 4,3 & 30,22 & 1,18 & 5,11 & 2,06 & 0,38 & 27,56 & 0,45 & 11,4 \\
\hline $0-2$ & 72,78 & 11,1 & 8,1 & 56,39 & 1,18 & 9,53 & 1,22 & 0,89 & 64,39 & 1,05 & 9,1 \\
\hline Total & 100,96 & & 14,4 & 100,00 & 1,18 & 16,91 & & 1,38 & 100,00 & 1,63 & 10,4 \\
\hline solo NF & 100 & & 16,8 & & 1,18 & 19,82 & & 1,61 & & 1,90 & 10,4 \\
\hline
\end{tabular}

NF: não fracionado 
Tabela 3. Amplitude das médias, do desvio padrão e do coeficiente de variação da porcentagem de massa e do teor de $\mathrm{C}$ e $\mathrm{N}$ das frações granulométricas, nos tratamentos A. prostrata e Capina, profundidades 0-10 e 10-20 cm.

\begin{tabular}{|c|c|c|c|c|c|}
\hline & \multicolumn{5}{|c|}{ Frações $(\mu \mathrm{m})$} \\
\hline & $200-2.000$ & $50-200$ & $20-50$ & $2-20$ & $0-2$ \\
\hline Média massa (\%) & $0,82-1,06$ & $8,34-8,40$ & $\begin{array}{c}0-10 \mathrm{~cm} \\
3,69-3,79\end{array}$ & $21,67-22,29$ & $66,20-67,30$ \\
\hline DP massa $\left(\mathrm{mg} \mathrm{g}^{-1}\right)$ & $0,12-0,26$ & $0,48-1,06$ & $0,29-0,64$ & $0,84-1,04$ & $0,62-1,32$ \\
\hline CV massa $(\%)$ & $11,6-31,4$ & $5,7-12,7$ & $7,8-16,9$ & $3,8-4,8$ & $0,9-2,0$ \\
\hline Média $C$ fração $\left(\mathrm{mg} \mathrm{g}^{-1}\right)$ & $63,3-96,1$ & $15,2-22,8$ & $12,6-15,4$ & $22,4-32,7$ & $12,3-14,6$ \\
\hline DP - C fração (mg g $\left.{ }^{-1}\right)$ & $11,7-30,3$ & $3,6-4,5$ & $0,9-4,2$ & $0,1-1,1$ & $0,16-0,21$ \\
\hline CV - C fração $(\%)$ & $12,2-47,8$ & $19,7-23,5$ & $7,5-27,5$ & $0,3-3,3$ & $1,3-1,4$ \\
\hline Média $\mathrm{N}$ fração $\left(\mathrm{mg} \mathrm{g}^{-1}\right)$ & $2,99-5,82$ & $1,06-1,76$ & $0,76-0,84$ & $2,14-2,93$ & $1,31-1,63$ \\
\hline DP - N fração (mg g $\left.{ }^{-1}\right)$ & $0,92-0,96$ & $0,25-0,30$ & $0,08-0,24$ & $0,05-0,26$ & $0,01-0,12$ \\
\hline CV - N fração (\%) & $16,5-31,0$ & $16,9-24,1$ & $\begin{array}{l}9,9-28,8 \\
10-20 \mathrm{~cm}\end{array}$ & $2,2-8,8$ & $1,0-7,3$ \\
\hline Média massa (\%) & $0,65-0,83$ & $7,36-7,97$ & $3,64-3,75$ & $20,81-23,72$ & $66,54-69,35$ \\
\hline DP - massa $\left(\mathrm{mg} \mathrm{g}^{-1}\right)$ & $0,10-0,14$ & $0,37-0,79$ & $0,20-0,31$ & $0,62-0,91$ & $0,86-0,99$ \\
\hline CV - massa (\%) & $12,5-21,1$ & $4,6-10,7$ & $5,5-8,4$ & $2,6-4,3$ & $1,2-1,5$ \\
\hline Média $\mathrm{C}$ fração $\left(\mathrm{mg} \mathrm{g}^{-1}\right)$ & $35-5-45,6$ & $12,4-14,0$ & $9,3-11,7$ & $22,9-24,7$ & $12,2-13,1$ \\
\hline DP - C fração $\left(\mathrm{mg} \mathrm{g}^{-1}\right)$ & $5,3-11,3$ & $0,7-2,4$ & $0,9-1,9$ & $0,4-0,5$ & $0,3-0,8$ \\
\hline CV - C fração (\%) & $11,7-31,9$ & $5,8-17,0$ & $10,4-16,2$ & $1,5-2,4$ & $2,3-6,4$ \\
\hline Média $N$ fração $\left(\mathrm{mg} \mathrm{g}^{-1}\right)$ & $1,44-2,19$ & $0,75-0,93$ & $0,55-0,75$ & $2,13-2,19$ & $1,34-1,44$ \\
\hline DP - N fração (mg g $\left.{ }^{-1}\right)$ & $0,09-0,33$ & $0,13-0,03$ & $0,07-0,13$ & $0,11-0,14$ & $0,04-0,12$ \\
\hline CV - N fração (\%) & $4,3-23,1$ & $3,9-13,5$ & $13,4-17,1$ & $4,8-6,5$ & $2,8-8,4$ \\
\hline
\end{tabular}

DP: desvio padrão; CV: coeficiente de variação.

massas das frações ocorreram nas frações areia grossa $(200-2.000 \mu \mathrm{m})$, concordando com os resultados obtidos por Feller et al. (1991). Isto se verificou, provavelmente pela pequena massa dessa fração (cerca de $1 \%$ ). Em conseqüência, o CV dos teores de $\mathrm{C}$ e $\mathrm{N}$ desta fração também foram altos. A mesma tendência foi observada nos compartimentos 50-200 e 20-50 $\mu \mathrm{m}$.

O balanço da massa de solo após a execução do fracionamento (Tabelas 1 e 2) indicou que houve uma recuperação de 96 a $105 \%$ da massa inicial, refletindo a adequada manipulação das amostras durante as análises (FREITAS et al., 2000; NEUFELDT; RESCK; AYARZA, 2002).

\section{Teores de C e N nas frações granulométricas}

$\mathrm{Na}$ fração areia grossa $(200-2.000 \mu \mathrm{m})$, os teores de C situaram-se entre 34,7 e 118,0 $\mathrm{mg} \mathrm{C} \mathrm{g}^{-1}$ de fração (Tabelas 1 e 2), valores próximos aos obtidos em oxisolos argilosos por Kouakoua (1998).
Desjardins et al. (1994) obtiveram 7,4 a 20,9 mg C g${ }^{1}$ de fração, para um latossolo de textura média. A pequena proporção de areia encontrada no solo do presente trabalho contribuiu para aumentar a proporção da parte orgânica na fração.

Na fração silte (2-20 $\mu \mathrm{m})$ os teores de $\mathrm{C}$ variaram de 18,2 a 36,1 $\mathrm{mg} \mathrm{C} \mathrm{g}^{-1}$ de fração (Tabelas 1 e 2). Em outros estudos foram obtidos valores superiores e com maior amplitude, de 31 a 106,0 $\mathrm{mg} \mathrm{C} \mathrm{g}^{-1}$ de fração (KOUAKOUA, 1998; FREITAS et al., 2000; FELLER, 1995).

Para a fração argila $(<2 \mu \mathrm{m})$, os teores de $\mathrm{C}$ variaram de 11,1 a $18,0 \mathrm{mg} \mathrm{C} \mathrm{g}^{-1}$ de fração, menores do que em outros trabalhos com 18,0 a 45,0 mg C g${ }^{1}$ de fração (KOUAKOUA, 1998; FREITAS et al., 2000; FELLER, 1995). A maior concentração de C encontra-se na fração argila para os solos podzólicos, luvisolos e terras roxas ácidas, enquanto nos chernozens encontra-se na fração silte fino (SCHULTEN; LEINWEBER, 1991). 
O teor de $\mathrm{N}$ (Tabelas 1 e 2) variou de 1,34 a 1,91 mg $\mathrm{N} \mathrm{g}^{-1}$ na fração argila e de 2,32 a 3,61 mg N g${ }^{-1}$ de fração silte, para todas as situações estudadas, sendo estes valores semelhantes aos encontrados por Freitas et al. (2000).

A contribuição de cada fração sobre o total de carbono revelou que, para a maioria das situações, a fração $<2 \mu \mathrm{m}$ foi a mais importante, sendo responsável por 35 a $51 \%$ do total, seguida da 2-20 $\mathrm{mm}$, com valores de 13 a $30 \%$ (Tabelas 1 e 2). Essas proporções são próximas das encontradas para condições de solos tropicais e subtropicais (FREITAS et al., 2000; SÁ et al., 2001), enquanto que sob clima temperado a proporção de $\mathrm{C}$ na fração argilosa vai de 35 a $70 \%$ (FELLER; BEARE, 1997).

O teor de C no solo para a fração 2-20 $\mu \mathrm{m}$ situouse entre 2,7 e 7,5 $\mathrm{mg} \mathrm{C} \mathrm{g}^{-1}$ solo e para a fração 0-2 $\mu \mathrm{m}$ foi de 7,7 a 11,0 $\mathrm{mg} \mathrm{C} \mathrm{g}^{-1}$ solo (Tabelas 1 e 2), concordando com as estimativas de Feller e Beare (1997), que observaram, ainda, que os teores de C nessas frações aumentam com o teor de argila do solo.

Para a relação C/N das frações, Feller (1993) observou que, de maneira geral, para a fração 200$2.000 \mu \mathrm{m}$ a relação $\mathrm{C} / \mathrm{N}$ foi $>15$, para a fração $2-20$ $\mu \mathrm{m}$ foi de $10-15$ e para a fração $<2 \mu \mathrm{m}$ foi $<10$, o que sugere que a $\mathrm{MO}$ associada às frações mais finas encontra-se em estágio mais avançado de decomposição do que a MO associada às frações maiores. Os dados registrados nesta pesquisa se situaram nestes valores (Tabelas 1 e 2), com exceção da fração 200-2.000 $\mu \mathrm{m}(0-10 \mathrm{~cm})$ sob floresta, que apresentou $\mathrm{C} / \mathrm{N}$ menor que $15(12,4)$.

\section{Efeito do manejo e do uso do solo sobre o conteúdo de $\mathrm{C}$ e a relação $\mathrm{C} / \mathrm{N}$ das frações}

Com a introdução das culturas, o decréscimo ocorrido no teor de $\mathrm{C}$ do solo foi proporcionalmente mais pronunciado nos compartimentos areia grossa + resíduos vegetais (200-2.000 $\mu \mathrm{m}$ ) e areia fina (50$200 \mu \mathrm{m}$ ), na camada $0-10 \mathrm{~cm}$ (Tabela 1 ). Na fração 200-2.000 $\mu \mathrm{m}$ o teor de C passou de 2,8 $\mathrm{mg} \mathrm{g}^{-1}$ de solo (na floresta) para 0,5-1,2 $\mathrm{mg} \mathrm{g}^{-1}$ de solo (em todas as outras situações de uso e manejo), o que representa perda de 48 a $81 \%$. Na fração 50-200 $\mu \mathrm{m}$ ocorreram perdas de 43 a $63 \%$. Nas frações silte $(2-20 \mu \mathrm{m})$ e argila $(<2 \mu \mathrm{m})$, as perdas foram de 8 a $30 \%$ e de 7 a $30 \%$. A maior perda do material orgânico das partículas da fração areia atribui-se ao material orgânico dessa fração que é composto principalmente por resíduos vegetais em estágios iniciais de decomposição e hifas de fungos, e portanto mais lábil, suscetível à oxidação e à desintegração pelo cultivo. Nas frações argila e silte a matéria orgânica é relativamente mais estável e a perda ocorre na massa microbiana e seus resíduos (DALAL; MAYER, 1986). Na camada 10-20 cm (Tabela 2), as diferenças entre a floresta nativa e as culturas não foram tão pronunciadas.

Em oxissolos tropicais, Feller (1993) observou que quando o solo sob vegetação natural foi submetido ao cultivo, as mudanças no estoque de carbono dependeram da textura do solo: para os arenosos a redução mais importante ocorreu na fração de resíduos vegetais (20-2.000 $\mu \mathrm{m})$ enquanto que para solos siltosos a redução foi devida principalmente às frações silte (2-20 $\mu \mathrm{m})$ e para os argilosos a redução do carbono ocorreu principalmente na fração argilosa, seguida da fração resíduo vegetal e depois silte. Entretanto, no presente trabalho observa-se que as maiores reduções no teor de $\mathrm{C}$ quando se compara a vegetação nativa com as culturas ocorreram nas frações 200-2.000 e 50-200 $\mu \mathrm{m}$, apesar do teor de argila ser de $691 \mathrm{~g} \mathrm{~kg}^{-1}$. Este fato também foi registrado por Martins et al. (1990), ao avaliarem a introdução de culturas após a retirada da mata em um latossolo amarelo podzolisado de textura média no Pará. Os autores observaram maiores reduções

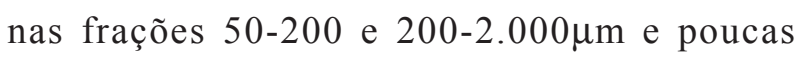
alterações na fração $<50 \mu \mathrm{m}$.

Em relação ao conteúdo de $\mathrm{C}$ e $\mathrm{N}$ do solo entre culturas anuais e o pomar vegetado com leguminosa, observa-se que a recuperação da $\mathrm{MO}$ do solo foi maior na fração 20-50 $\mu \mathrm{m}$, pois essa fração não só 
atingiu o teor inicial, como ficou $26 \%$ acima do teor da floresta para o C e $10 \%$ acima para o $\mathrm{N}$, na camada de $0-10 \mathrm{~cm}$ (Tabela 1 ).

\section{Conclusões}

Com a introdução das culturas, as maiores perdas de $\mathrm{C}$ e $\mathrm{N}$ ocorreram nas frações granulométricas 200-2.000 e 50-200 $\mu \mathrm{m}$, as quais correspondem a frações orgânicas em estágios iniciais de decomposição.

Os sistemas de manejo do solo do pomar que mais contribuíram na recuperação dos teores de $\mathrm{C}$ e $\mathrm{N}$ do solo foram com plantas de cobertura (leguminosa ou vegetação espontânea mantida com grade/roçadora).

Analisando-se o solo sob culturas anuais e o pomar com vegetação de leguminosa, a fração 20-50 $\mu \mathrm{m}$ foi a que apresentou maior aumento da matéria orgânica.

\section{Agradecimentos}

À CAPES/MEC, pela oportunidade de realização do trabalho por meio de Bolsa Doutorado Sanduíche.

\section{Referências}

BALESDENT, J.; PETRAUD, J.P.; FELLER, C. Effets des ultrasons sur la distribution granulométrique des matières organiques des sols. Science du Sol, Paris, v.29, n.2, p.95106, 1991.

CERRI, C.; FELLER, C.; BALESDENT, J.; VICTORIA, R.; PLENECASSAGNE, A. Application du traçage isotopique naturel en $13 \mathrm{C}$, à l'éude de la dynamique de la matière organique dans les sols. Comptes Rendus de l'Académie des Sciences, Paris, v.300, n.9, p.423-428, 1985. série 2.

CERRI, C. C.; FELLER, C.; CHAUVEL, A. Evolução das principais propriedades de um Latossolo Vermelho-Escuro após desmatamento e cultivo por doze e cinqüenta anos com cana-de-açúcar. Cahiers ORSTOM: Serie Pedologie, Bondy, v.26, p. 37-50, 1991.

CHRISTENSEN, B.T. Physical fractionation of soil orgaic matter in primary particle size and density separates. Advances in Soil Science, New York, v.20, p.1-19, 1992.
DALAL, R.C.; MAYER, R. J. Long-term trends in fertility of soils under continuous cultivation and cereal cropping in southern Queenland. III. Distribution and kinetics of soil organic carbon in particle-size fractions. Australian Journal of Soil Research, Melbourne, v.24, n.2, p.293-300, 1986.

DESJARDINS, T.; ANDREUX, F.; VOLKOFF, B.; CERRI, C. Organic carbon and ${ }^{13} \mathrm{C}$ contents in soils and soil sizefractions, and their changes due to deforastation and pasture installation in eastern Amazonia. Geoderma, Amsterdam, v.61, n.1/2, p.103-118, 1994.

EMBRAPA. Manual de métodos de análise de solo. Rio de Janeiro: SNLCS, 1979.

FEIGL, B.J.; MELILLO, J.; CERRI, C.C. Changes in the origin and quality of soil organic matter after pasture introduction in Rondônia (Brazil). Plant and Soil, The Hague, v. 175, n.1, p. 21-29, 1995.

FELLER, C.; BURTIN, G.; GERARD, B.; BALESDENT, J. et al.? et al. Utilisation des résines sodiques et des ultrasons dans le fractionnement granulométrique de la matière organique des sols, intérêt et limites. Science du Sol, Paris, v.29, n.2, p.77-93, 1991.

FELLER, C. La matière organique dans les sols tropicaux à argile 1: 1; recherche de compartiments organiques fonctionnels: une approche granulométrique. Paris: ORSTOM, 1995. (Coll. Tdm-ORSTOM, 144).

FELLER, C. Organic inputs, soil organic matter and functional soil organic compartments in low-activity clay soils in tropical zones. In: MULONGOY, K.; MERCKX, R. (Ed.). Soil organic matter and sustainability of tropical agriculture. Chichester: Willey-Sayce, 1993. p.77-88.

FELLER, C.; BEARE, M.H. Physical control of soil organic matter dynamics in the tropics. Geoderma, Amsterdam, v.79, n.1-4, p.69-116, 1997.

FREITAS, P.L.; BLANCANEAUX, P.; GAVINELLI, E.; LARRÉ-LARROUY, M.C.; FELLER, C. Nível e natureza do estoque orgânico de latossolos sob diferentes sistemas de uso e manejo. Pesquisa Agropecuária Brasileira, Brasília, v.35, n.1,p.157-171, 2000.

FREIXO, A.A.; MACHADO, P.L.O.A.; GUIMARÃES, C.M.;SILVA, C.A.; FADIGAS, F.S. Estoques de carbono e nitrogênio e distribuição de frações orgânicas de latossolo do cerrado sob diferentes sistemas de cultivo. Revista Brasileira de Ciência do Solo, Viçosa, v.26, p.425-434, 2002.

GAVINELLI, E.; FELLER, C.; LARRÉ-LARROUY, M. C.; BACYE, B.; DJEGUI, N. A routine method to study soil organic matter by particle size fractionation: examples for tropical soils. Communications in Soil Science and Plant Analysis, New York, v.26, n.11/12, p.1749-1760, 1995. 
GOMES, F.P. A estatística moderna na pesquisa agropecuária. Piracicaba: POTAFOS, 1987.

KOUAKOUA, E. La matière organique et la stabilité structurale d'horizons de surface de sols ferrallitiques argileux, effet du mode de gestion des terres. 1998. Thèse (Docteur em Science du Sol) - Faculté de Sciences, Université Henri Poincaré, Nancy 1.

LAL, R. Residue management, conservation tillage and soil restoration for mitigating greenhouse effect by $\mathrm{CO} 2-$ enrichment. Soil \& Tillage Research, Amsterdam, v.43, n.1, p.81-107, 1997.

LEPSCH, I.F.; SILVA, N.M ; ESPIRONELO, A. Relação entre matéria orgânica e textura de solos sob cultivo de algodão e cana-de-açúcar, no Estado de São Paulo. Bragantia, Campinas, v.41, p.231-236, 1982.

MARTINS, P.F.S.; CERRI, C.C.; VOLKOFF, B.; ANDREUX, F. Conseqüências do cultivo e do pousio sobre a matéria orgânica do solo sob floresta natural na Amazônia oriental. Acta Amazonica, Manaus, v. 20, p.1828, 1990 .

NEUFELDT, H.; RESCK, D.V.S.; AYARZA, M.A. Texture and land-use effects on soil organic matter in cerrado oxisols, central Brazil. Geoderma, Amsterdam, v.107, n.34,p.151-164, 2002.

NEVES, C. S. V.J.; DECHEN, A. R.; FELLER, C.; GONZALEZ, M. G. N. Influência de sistemas de manejo de solo em pomar de tangerina 'Poncã' sobre limão 'Cravo' em um latossolo roxo. Revista Brasileira de Fruticultura, Cruz das Almas, v.20, n.3, p.367-374, 1998.
ROSCOE, R.; BUURMAN P.; VELTHORST, E.J. Disruption of soil aggregates by varied amounts of ultrasonic energy in fractionation of organic matter of a clay latosol: carbon, nitrogen and delta ${ }^{13} \mathrm{C}$ distribution in particle-size fractions. European Journal of Soil Science. v.51, n.3, p.445-454, 2000.

SÁ, J.C.M.; CERRI, C.C. R.; DICK, W. A.; LAL, R.; VENSKE FILHO, S. P. PICCOLO, M. C.; FIEGL, B. E. Organic matter dynamics and carbon sequestration rates for a tillage chronosequence in a Brazilian Oxisol. Soil Science Society of America Journal, Madison, v.65, n.5, p.1486-1499, 2001.

SÁ, J. C. M. Manejo da fertilidade do solo no plantio direto. Castro: ABC, 1993.

SCHNITZER, M. Binding of humic substances by mineral colloids. In: HUANG, P.M.; SCHNITZER, M (Ed.). Interactions of soil minerals with natural organics and microbes. Wisconsin: SSSA, 1986. p.77-101.

SCHULTEN, H. R.; LEINWEBWER, P. Influence of longterm fertilization with farmyard manure on soil organic matter: characteristics of particle-size fractions. Biology and Fertility of Soils, Heidelberg, v.12, n.2, p.81-88, 1991.

VAUGHAN, D.; ORD, B. G. Soil organic matter - a perspective on its nature, extraction, turnover and role in soil fertility. In: VAUGHAN, D.; MALCOLM, R. E. Soil organic matter and biological activity. Dordrecht: Martinus Nijhoff, 1985. p.1-35. 